\title{
ON COVERINGS OF CONVEX SETS BY TRANSLATES OF SLABS
}

\author{
H. GROEMER ${ }^{1}$
}

\begin{abstract}
Let $\left(S_{1}, S_{2}, \ldots\right)$ be a sequence of slabs in euclidean $n$-dimensional space and let $t_{i}$ denote the thickness of $S_{i}$. It is shown that the condition $\sum t_{i}^{(n+1) / 2}=\infty$ implies that every convex set can be covered by translates of the slabs $S_{i}$, and that the exponent $(n+1) / 2$ is, in a certain sense, best possible.
\end{abstract}

1. A closed subset of euclidean $n$-dimensional space $R^{n}$ is called a slab of thickness $t$ if its boundary consists of two parallel hyperplanes having mutual distance $t$. If $K$ is a convex subset of $R^{n}$ and $S$ a slab we say that $K$ is not dissected by $S$ if $K \backslash S$ is again convex. Let now $C$ be a compact convex subset of $R^{n}$ and $\left(S_{i}\right)=\left(S_{1}, S_{2}, \ldots\right)$ a sequence of slabs in $R^{n}$. Then $\left(S_{i}\right)$ is said to permit a translative covering of $C$ if there are translates $S_{1}^{\prime}, S_{2}^{\prime}, \ldots$ of $S_{1}, S_{2}, \ldots$ such that $C \subset \cup S_{i}^{\prime}$. Moreover, $\left(S_{i}\right)$ is said to permit a nondissecting translative covering of $C$ if, in addition, $C$ is not dissected by $S_{1}^{\prime}, C \backslash S_{1}^{\prime}$ is not dissected by $S_{2}^{\prime}$, $C \backslash\left(S_{1}^{\prime} \cup S_{2}^{\prime}\right)$ is not dissected by $S_{3}^{\prime}$, and so on.

Let $t_{i}$ denote the thickness of $S_{i}$. We concern ourselves with the problem of finding conditions on the sequence $\left(t_{i}\right)$ in order that $\left(S_{i}\right)$ permits a nondissecting translative covering of a given (or of every) compact convex set in $R^{n}$. It is easy to see that the condition $\Sigma t_{i}^{n}=\infty$ is sufficient. For $n=2$ it has been shown in [1] that $\sum t_{i}^{3 / 2}=\infty$ is already sufficient and that the exponent $3 / 2$ is essentially best possible. But the method used there does not generalize to arbitrary $n$. We are now using a different method, involving the parallel bodies of the pertinent convex sets, to prove for all $n \geqslant 1$ similar results as in [1]. The condition $\Sigma t_{i}^{3 / 2}=\infty$ will appear as a special case of the corresponding condition for $R^{n}$, namely $\Sigma t_{i}^{(n+1) / 2}=\infty$.

If not only translations but arbitrary rigid motions are permitted the analogous problem leads of course immediately to the famous "plank problem" of Tarski. It should also be noted that the concept of a nondissecting translative covering depends on the particular ordering of the slabs in the given sequence. If rearrangements are permitted the corresponding problems are of a rather different nature. Various problems and results related to the present paper are discussed in [1] and in the literature listed there.

2. If $C$ is a compact convex subset of $R^{n}$ we denote by $W_{0}(C)$, $W_{1}(C), \ldots, W_{n}(C)$ the mean projection measures (Quermassintegrale) of $C$. The

Received by the editors August 13, 1980.

1980 Mathematics Subject Classification. Primary 52A45.

${ }^{1}$ Supported by National Science Foundation Research Grant MCS-8001578.

(c) 1981 American Mathematical Society 0002-9939/81/0000-0270/\$02.50 
volume of the $n$-dimensional unit ball will be denoted by $\kappa_{n}$. Using these notations we can formulate our principal result.

THeOREM 1. For $n>1$ let $C$ be a compact convex subset of $R^{n}$ and $\left(S_{1}, S_{2}, \ldots\right)$ a (finite or infinite) sequence of slabs in $R^{n}$. Let $t_{i}$ denote the thickness of $S_{i}$, and assume that $t_{i} \leqslant 1$ (for all $i$ ). Then, $\left(S_{i}\right)$ permits a nondissecting translative covering of $C$ if

$$
\sum_{i} t_{i}^{(n+1) / 2}>\frac{n}{\kappa_{n-1}}\left(W_{0}(C)+\left(\begin{array}{c}
n \\
1
\end{array}\right) W_{1}(C)+\cdots+\left(\begin{array}{c}
n \\
n-1
\end{array}\right) W_{n-1}(C)\right) .
$$

In the case $n=2$ we denote by $a(C)$ the area and by $p(C)$ the perimeter of $C$. Then we have $a(C)=W_{0}(C), p(C)=2 W_{1}(C)$ and it follows that the condition $\Sigma t_{i}^{3 / 2}>a(C)+p(C)$ is sufficient for the existence of a nondissective translative covering of a compact convex domain $C$ in $R^{2}$ by strips. This statement is slightly stronger than the main theorem in [1].

The following corollary is an immediate consequence of Theorem 1.

COROLlaRY 1. If $\left(S_{i}\right)$ is a sequence of slabs in $R^{n}$ and $t_{i}$ denotes the thickness of $S_{i}$, then $\left(S_{i}\right)$ permits a nondissecting translative covering of every compact convex subset of $R^{n}$ if

$$
\sum_{i=1}^{\infty} t_{i}^{(n+1) / 2}=\infty .
$$

In this corollary it is not necessary to include the condition $t_{i}<1$. This can easily be seen as in [1] by considering separately the cases when infinitely many or only finitely many slabs of thickness greater than 1 appear.

As another consequence of Theorem 1 we can state the following result concerning coverings of all $R^{n}$.

COROLlary 2. If $\left(S_{i}\right)$ is a sequence of slabs in $R^{n}$ and $t_{i}$ denotes the thickness of $S_{i}$ then $\left(S_{i}\right)$ permits a translative covering of $R^{n}$ if $\sum_{i=1}^{\infty} t_{i}^{(n+1) / 2}=\infty$.

Concerning the question whether the exponent $(n+1) / 2$ in Theorem 1 or Corollary 1 is best possible, we can prove the following theorem.

TheOREM 2. For every $n>1$ there exists a sequence $\left(S_{i}\right)$ of slabs in $R^{n}$ with the following properties:

(i) If $t_{i}$ is the thickness of $S_{i}$ then $t_{i}<1$ and $\sum_{i=1}^{\infty} t_{i}^{\alpha}=\infty$ for all $\alpha<(n+1) / 2$.

(ii) $\left(S_{i}\right)$ does not permit a nondissecting translative covering of the $n$-dimensional unit ball.

This theorem shows that neither in Theorem 1 nor in Corollary 1 is it possible to replace the exponent $(n+1) / 2$ by any smaller number, and that this remains to be true even if the right-hand side of (1) is replaced by any other real valued function on the class of all compact convex subsets of $R^{n}$. Since Corollary 2 is not dealing with nondissecting coverings, Theorem 2 does not apply in this situation, and it is quite likely that improvements are possible for all $n>1$. Actually, for $n=2$ it has already been shown by P. Erdös and E. G. Straus that for such coverings the condition $\Sigma t_{i}^{3 / 2}=\infty$ can be replaced by $\Sigma t_{i}=\infty$. 
3. We turn now to the proof of Theorem 1. $K$ will always denote a bounded convex subset of $R^{n}$ of volume $v(K)$. By $B(r)$ we denote the $n$-dimensional ball of radius $r$ and with center at the origin $o$ of $R^{n}$; but instead of $B(1)$ we write simply $B$. The parallel body of $\mathrm{cl} K$ at distance 1 will be denoted by $P(K)$. Hence, $P(K)=($ cl $K)+B$. A slab $S$ is said to be parallel to a hyperplane $E$, or to a half-space with boundary plane $E$, if the boundary planes of $S$ are parallel to $E$. We note that

$$
v(P(K))<\kappa_{n} \text { implies } K=\varnothing .
$$

If $H$ is a closed half-space in $R^{n}$ it determines a dissection of $K$ into two "sections" $K_{H}^{+}, K_{H}^{-}$which are defined by $K_{H}^{+}=K \cap H, K_{H}^{-}=K \backslash(K \cap H)$. The slab that contains $K_{H}^{-}$and is parallel to $H$ and of minimal thickness will be denoted by $S_{H}(K)$, and the thickness of $S_{H}(K)$ by $d_{H}(K)$. If $H$ has the property that $d=d_{H}(B)$ $<1$ then

$$
v\left(B_{H}^{-}\right) \geqslant \frac{\kappa_{n-1}}{n} d^{(n+1) / 2} .
$$

This follows from the obvious fact that it is possible to inscribe in $\operatorname{cl} B_{H}^{-}$a cone which has height $d$ and an $(n-1)$-dimensional ball of radius $\sqrt{1-(1-d)^{2}}$ $>\sqrt{d}$ as base. More generally, if $K \neq \varnothing$ and $d_{H}(P(K))<1$ then it follows from the definition of $P(K)$ that there is a unit ball (which may be assumed to be $B$ ) such that $B \subset P(K)$ and $d_{H}(P(K))=d_{H}(B)$. Using (3) we can therefore state that the condition $d=d_{H}(P(K))<1$ implies

$$
v\left((P(K))_{H}^{-}\right) \geqslant \frac{\kappa_{n-1}}{n} d^{(n+1) / 2} .
$$

If $G$ is a closed half-space with $G \subset H$ and such that bdr $G$ and bdr $H$ are parallel and have mutual distance 1 then

$$
d_{H}(P(K))=d_{G}(K)
$$

and

$$
P(K \cap G) \subset P(K) \cap H .
$$

(5) holds since the distance between a supporting plane of $K$ and a supporting plane of $P(K)$, both planes having the same direction, is 1 . Relation (6) is true since any point of $P(K \cap G)$ has distance at most 1 from $K$ and also from $G$, and is therefore both in $P(K)$ and in $H$. (6) implies that

$$
v\left(P\left(K_{G}^{+}\right)\right)<v\left((P(K))_{H}^{+}\right)=v(P(K))-v\left((P(K))_{H}^{-}\right) .
$$

If $d=d_{G}(K)<1$ we deduce from (4), (5) and (7) that

$$
v\left(P\left(K_{G}^{+}\right)\right)<v(P(K))-\frac{\kappa_{n-1}}{n} d^{(n+1) / 2} .
$$

If $C ; S_{1}, S_{2}, \ldots ; t_{1}, t_{2}, \ldots$ are defined as in Theorem 1 and (1) is satisfied we are now describing a procedure for covering $C$. Without any loss of generality we may assume that int $S_{i} \neq \varnothing$ for all $i$.

First, we find a translate $S_{1}^{\prime}$ of $S_{1}$ such that either $C \subset S_{1}^{\prime}$ or, when this is not possible, so that one of the boundary planes of $S_{1}^{\prime}$ is a supporting plane of $C$ and 
$C \cap$ int $S_{1}^{\prime} \neq \varnothing$. In the first case, a nondissecting translative covering of $C$ has been found. In the second case $S_{1}^{\prime}$ does not dissect $C$, and if we define $C_{1}=$ $C \backslash S_{1}^{\prime}$, there is a half-space, say $H_{1}$, so that $S_{H_{1}}(C)=S_{1}^{\prime}$ and therefore $d_{H_{1}}(C)=$ $t_{1}$. It follows from (8) (take $K=C, G=H_{1}, d=t_{1}$ ) that

$$
v\left(P\left(C_{1}\right)\right)<v(P(C))-\frac{\kappa_{n-1}}{n} t_{1}^{(n+1) / 2} .
$$

Similarly, we can take $C_{1}$ instead of $C$, and $S_{2}$ instead of $S_{1}$. Then, we find a translate $S_{2}^{\prime}$ of $S_{2}$ which yields either a nondissecting translative covering of $C_{1}$, and therefore a corresponding covering of $C$ by $S_{1}^{\prime} \cup S_{2}^{\prime}$, or a convex set $C_{2}=$ $C_{1} \backslash S_{2}^{\prime}=C \backslash\left(S_{1}^{\prime} \cup S_{2}^{\prime}\right)$ with

$$
v\left(P\left(C_{2}\right)\right)<v\left(P\left(C_{1}\right)\right)-\frac{\kappa_{n-1}}{n} t_{2}^{(n+1) / 2} .
$$

From (9) and (10) it follows that

$$
v\left(P\left(C_{2}\right)\right) \leqslant v(P(C))-\frac{\kappa_{n-1}}{n}\left(t_{1}^{(n+1) / 2}+t_{2}^{(n+1) / 2}\right) .
$$

If $k$ repetitions of this procedure are possible, that means if no covering of $C$ has been obtained at a previous step, we find translates $S_{1}^{\prime}, S_{2}^{\prime}, \ldots, S_{k}^{\prime}$ of $S_{1}$, $S_{2}, \ldots, S_{k}$ so that $C_{k}=C \backslash\left(S_{1}^{\prime} \cup S_{2}^{\prime} \cup \cdots \cup S_{k}^{\prime}\right)$ is convex and

$$
v\left(P\left(C_{k}\right)\right)<v(P(C))-\frac{\kappa_{n-1}}{n} \sum_{i=1}^{k} t_{i}^{(n+1) / 2} .
$$

Since Steiner's formula for parallel bodies shows that

$$
v(P(C))=\sum_{j=0}^{\infty}\left(\begin{array}{l}
n \\
j
\end{array}\right) W_{j}(C)
$$

we deduce from (1) and (11) that $v\left(P\left(C_{k}\right)\right)<W_{n}(C)$, provided that $k$ is large enough. Because of $W_{n}(C)=\kappa_{n}$ this shows that $v\left(P\left(C_{k}\right)\right)<\kappa_{n}$ and, taking into account (2), we find $C_{k}=\varnothing$. Hence, the finite sequence $\left(S_{1}^{\prime}, S_{2}^{\prime}, \ldots, S_{k}^{\prime}\right)$ provides already a covering of $C$ of the desired kind.

4. For the proof of Theorem 2 we may obviously assume that $n>2$. We use the same notations as in the proof of Theorem 1. If $H$ is a half-space the set $B(r)_{H}^{-}$will be called a spherical section of radius $r$ and thickness $d_{H}(B(r))$. A class $\left\{B(r)_{H_{1}}^{-}, B(r)_{H_{2}}^{-}, \ldots, B(r)_{H_{m}}^{-}\right\}$of spherical sections will be called symmetric if the set $\cup_{i=1}^{m} B(r)_{H_{i}}^{-}$is symmetric with respect to $o$. We need the following auxiliary result.

LEMMA. For every $n>2$ there exists a constant $c_{n}$ so that for all $r \geqslant \frac{1}{2}, 0<t<r$, one can find a symmetric set of more than $c_{n} t^{(1-n) / 2}$ disjoint spherical sections of radius $r$ and thickness $t$.

Proof. We assume first that $r=1$. If $0<t<1$ and $T$ is a spherical section of radius 1 and thickness $t$ let $\beta_{t}$ denote the geodesic radius of the spherical cap $T \cap$ bdr $B$. Since the lemma is trivial under the assumption $\beta_{t}>\pi / 4$ we may suppose that $\beta_{t} \leqslant \pi / 4$. Under this restriction it can be shown that there exists a 
constant $a_{n}$ such that there are $m$ disjoint spherical sections of radius 1 and thickness $t$ so that

$$
m \int_{0}^{2 \beta_{t}}(\sin x)^{n-2} d x>a_{n}
$$

A short proof of this can be found in [2] (symmetry is not mentioned there but can obviously be added). As an immediate consequence of (12) we obtain

$$
m \beta_{t}^{n-1}>2^{n-1}(n-1) a_{n} .
$$

Now we note that $t=1-\cos \beta_{t}>\frac{1}{2} \sin ^{2} \beta_{t}$ and that the assumption $\beta_{t}<\pi / 4$ implies (due to the monotonicity of $\sin x / x) \sin \beta_{t}>(2 \sqrt{2} / \pi) \beta_{t}$. It follows that $t>\left(4 / \pi^{2}\right) \beta_{t}^{2}$, and this enables us to deduce from (13) that $m>b_{n} t^{(1-n) / 2}$ with some constant $b_{n}$. If we assume only that $r>\frac{1}{2}$, rather than $r=1$, we can perform a similarity transformation and obtain $m>b_{n} r^{(n-1) / 2} t^{(1-n) / 2}>b_{n} 2^{(1-n) / 2} t^{(1-n) / 2}$. Hence the lemma holds with $c_{n}=b_{n} 2^{(1-n) / 2}$.

We now give the proof of Theorem 2. It is modeled after the proof for $n=2$ in [1]. Let $\sum_{j=1}^{\infty} p_{j}$ be an infinite series with the properties that $p_{j}>0$,

$$
\sum_{j=1}^{\infty} p_{j}<\frac{1}{2}
$$

and

$$
\sum_{j=1}^{\infty} p_{j}^{\sigma}=\infty \quad \text { for every } \sigma<1 .
$$

For example, $\sum_{j=1}^{\infty} 1 /(j+k) \log ^{2}(j+k)$ has this property if $k$ is large enough.

If the above lemma is applied to the case $r=1, t=p_{1}$ we obtain a symmetric set of an even number, say $2 m_{1}$, of disjoint spherical sections $B_{H_{1}}^{-}, B_{H_{2}}^{-}, \ldots, B_{H_{2 m_{1}}}^{-}$ where $d_{H_{i}}(B)=p_{1}$ and $2 m_{1}>c_{n} p_{1}^{(1-n) / 2}$. Moreover, it may be assumed that the enumeration is such that $B_{H_{i}}^{-}=-B_{H_{i+m_{1}}}^{-}\left(i=1,2, \ldots, m_{1}\right) . S_{1}, S_{2}, \ldots, S_{m_{1}}$ are now defined as the slabs with the property that $S_{i}=S_{H_{i}}(B)$. Next we define $m_{2}$ slabs $S_{m_{1}+1}, \ldots, S_{m_{1}+m_{2}}$ by the analogous procedure, taking $r=1-p_{1}, t=p_{2}$, and continue accordingly. Then, the slabs

$$
S_{m_{1}}+\cdots+m_{k-1}+1, S_{m_{1}}+\cdots+m_{k-1}+2, \ldots, S_{m_{1}}+\cdots+m_{k-1}+m_{k}
$$

have all equal thickness $p_{k}$ and

$$
m_{k}>c_{n} p k^{(1-n) / 2} / 2 \text {. }
$$

We note also that the ball that is used in defining these slabs has radius $1-p_{1}$ $-\cdots-p_{k-1}$, where (because of (14))

$$
1-p_{1}-\cdots-p_{k-1}>\frac{1}{2} \text {. }
$$

If $t_{i}$ denotes the thickness of $S_{i}$ (i.e. $t_{1}=t_{2}=\cdots=t_{m_{1}}=p_{1}, t_{m_{1}+1}=t_{m_{1}+2}$ $=\cdots=t_{m_{1}+m_{2}}$, etc.) and if $\alpha<(n+1) / 2$ we deduce from (16) that

$$
\sum_{i=1}^{\infty} t_{i}^{\alpha}=\sum_{k=1}^{\infty} m_{k} p_{k}^{\alpha} \geqslant \frac{c_{n}}{2} \sum_{k=1}^{\infty} p_{k}^{(1-n) / 2} p_{k}^{\alpha}=\frac{c_{n}}{2} \sum_{k=1}^{\infty} p_{k}^{\sigma}
$$


where $\sigma=(1-n) / 2+\alpha<1$. Hence, it follows from (15) and (18) that $\sum t_{i}^{\alpha}=\infty$. This, together with the fact that (14) implies $t_{i}<1$, shows that part (i) of Theorem 2 is true.

Finally, to prove part (ii) we assume that $S_{1}^{\prime}, S_{2}^{\prime}, \ldots$ are translates of $S_{1}, S_{2}, \ldots$ such that $B$ is not dissected by $S_{1}^{\prime}, B /\left(S_{1}^{\prime} \cup \cdots \cup S_{i}^{\prime}\right)$ is not dissected by $S_{i+1}^{\prime}$ $(i=1,2, \ldots)$ and prove that $\cup S_{i}^{\prime}$ does not cover $B$. The construction of $S_{1}$ shows that $S_{1}^{\prime}$ has no interior points in common with $B_{H_{1}}^{+} \cap B_{-H_{1}}^{+}$. Since $\left(B_{H_{1}}^{-} \cup\right.$ $\left.B_{-H_{1}}^{-}\right) \cap\left(B_{H_{2}}^{-} \cup B_{-H_{2}}^{-}\right)=\varnothing$ it is also clear that $S_{2}^{\prime}$ has no interior points in common with $B_{H_{2}}^{+} \cap B_{-H_{2}}^{+}$, and so on up to $S_{m_{1}}^{\prime}$. Since each of the sets $B_{H_{i}}^{+} \cap B_{-H_{1}}^{+}(i=$ $\left.1,2, \ldots, m_{1}\right)$ contains the ball $B\left(1-t_{1}\right)$ it follows that none of the slabs $S_{1}^{\prime}, \ldots, S_{m_{1}}^{\prime}$ has interior points in common with $B\left(1-t_{1}\right)$. In an analogous manner one finds that the slabs $S_{m_{1}+1}^{\prime}, S_{m_{1}+2}^{\prime}, \ldots, S_{m_{1}+m_{2}}^{\prime}$ have no interior points in common with $B\left(1-t_{1}-t_{2}\right)$, and a continuation of this procedure shows that none of the slabs $S_{i}^{\prime}(i=1,2, \ldots)$ has interior points in common with $B\left(1-\sum_{i=1}^{\infty} t_{i}\right)$. Since (17) implies that $B\left(\frac{1}{2}\right) \subset B\left(1-\sum_{i=1}^{\infty} t_{i}\right)$ it is clearly impossible that $\cup_{i=1}^{\infty} S_{i}^{\prime}$ covers $B$.

\section{REFERENCES}

1. H. Groemer, On coverings of plane convex sets by translates of strips, Aequationes Math. (to appear).

2. A. D. Wyner, Capabilities of bounded discrepancy decoding, Bell System Tech. J. 44 (1965), 1061-1122. MR 31, 4652.

Department of Mathematics, University of Arizona, Tucson, Arizona 85721 